\title{
-N84 27274
}

THEORY AFD MRASURE IF CERTAIN IMAGE NORAS IN SAR

\author{
R. Keith kiney \\ RADAPSAT \\ Canada Centre for remote Sensing \\ ottawa, Canada
}

\begin{abstract}
This paper summarizes principal properiles of SAR imagery of point and distributed objects. Against this background, the response of a SAR (Synthetic Aperture Radar) to the moving surface of the sea is considered. Certain conclusions are drawn as to the mechanism of interaction between microwaves and the sea surface.

It. has been estajlished for a "well-hehaved" SAR (as for other radars) that a principle of conservation of energy is satisfied. This means that the energy of the response (in the image) is constant under conditions of cilangling phase of the signal. Phase errors may arise systematically, such as focus mis-match to parameters appropriate to a specular scattering centre, or randomly, as from complex motion of the sea surface. Of course, focus errors reduce the peak and spread the impulse response of the image of a point target. Focus errors do not, however, change the speckle spectrum of a truly random "uniform" Gaussian scene.

Focus and speckle spectral tesis may be used on selected SAR 1magery for areas of the ocean. When this is done, it is observed that the fine structure of the sea imagery is sensitive to processor focus and adjustment. Furthermore, there is frequently correlation between nominally statisticly independent looks. Therefore, the ocean reflectivity mechanism must include point-like scatterers of sufficient radar cross-section to dominate the return from certain individual resolution elements. Furthermore, both specular and diffuse scattering mechanisms are observed together, to varying degree. The effect is sea state dependent, of course. This mechanism would explain the evident diversity of theoretical opinion on the subject of SAR wave response.
\end{abstract}

Several experiments are proposed based on Imaging theory that could assist in the investigation of reflectivity mechanims. 


\section{INTRONUCTION}

It is a reasonable requirement of SAR (Synthetic Aperture Radar) ocean reflectivity theories that they be consistent in every regard with the facts of life of SAR image formation. To the knowledge of this author, there are no theorles of ocean reflectlvity that pass this test.

A SAR, even operating in a partially coherent mode, is a special kind of Iinear system. Several fundamental properties for such systems have been rigourously proven (Harger, 1970, Raney, 1983). The observation by the SAR of particular scenes, such as an ocean surface, cannot change these facts.

The purpose of this peper is two-fold. First, pertinent properties of SAR operation are presented and succinctly discussed. Whereas many of these properties have been known for some years, they seem not to have been fully apprehended by workers in the ocean reflectivicy field. Second, there follow from the first discussion consequences of importance to the oceanographic application. Several of these are highlighted and discussed. Furthermore, it is posstble to design certain data processing and field experiments that may be used to take advantage of these properties, and so to shed some light on the reflectivity mechanisms involved. Suggestions are made for such tests.

The major thrust of Raney (1983) is that for SAR (as for all radar systems) there exist fundamental properties such as conservation of energy. These propertles, if suitably employed, can be helpful in deriving quantitative information about the reflectivity mectanism from the imagery. The matter is complicated by (1) the partial coherence of most radar systems, (2) non-1inearities and temporal variations found in all practical radar systems, and (3) the presence of both specular and diffuse scatterers in the input scene. This work deals directly with (1), disregards (2), and makes some observations based on (3) that have direct impact on SAR reflectivity models of the ocean surface.

Following the introduction, Section 2 of the paper considers sultable model representation of a SAR. Basic properties are identified in signal processing considerationz.

Section 3 considers the "impulse response" of a SAR, the way in which the system images an idealized point object. Section 4 reviews the way in which a SAR Images an Idealized uniform random distributed scene such as the classic wheat field. In each of these discussions, radar system parameters (such as nominal resolution and bandwidth) and processing system parameters (such as focus and mult1-looking) are considered.

In Section 5 basic propertles of speckle are revlewed. As is well known, radar speckle 18 an unwelcome but ever present characteristic of quas1-coherent imagery, and $1 t$, too, obeys certa1n well established princtples. 
In Section 6, the properties previously presented are revisited, with the objective of seeing them from the point of view of applications to the oceanic imaging problem, leading to a discussion of recommended experiments. The paper has a brief concluding section.

\section{SAR MODEL}

The baslc objectlve of an imagling radar system is deceptiveiy simple: we wish to derlve an "1mage" which is a mapping of the reflectivity of a scene observed by microwave probing of the real world, expressed in photographic form. In model language, real world reflectivity $\delta_{0}(x, y)$ is estimated as $\hat{\sigma}_{0}(x, y)$ through a microwave transducer (MT) thus

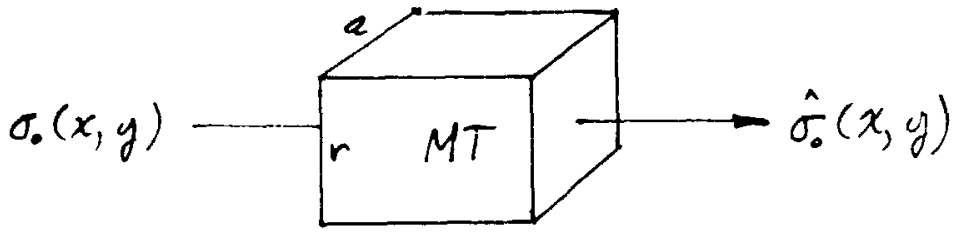

... and at the outset, complexities are apparent. Let us confine the discussion to synthectc aperture radar (SAR) systema. Then the following are true:

1) SAR systems "work" because of the different mechanisms used to form the azimuth (a) and the range ( $r$ ) dimensions of the image (Harge:, 1970 ). Range scanning is at one half the speed of light (thus in effect instantaneous) and continuous. Azimuth scanning is at the speed of the carrier vehicle (thus at velocitles sensitive to possible chariges in the scene itself) and is dependent on the pulsed nature of the radar. For the moment, we ignore these fundamental range and azimuth differences, and treat the two "channeis" of a SAR in like manner, a salisfactory approximation for the first sections of this paper. The differences in time scale between these channels 1ie at the core of the SAR ocean Imaging problem, however.

11) The transducer "MT" is not perfect. That is, it is not able to Image all of the detall inherent in the scene. This characteristic 1s typical of any lmaging system: resolution (In range or azimuth) 18 a measure of this limitation. There is rather 11ttle subtlety in this consideration, except that...

111) The microwave probe (transmitted signal of the SAR) is essential1y monochromatic and coherent. There are many important Implications that follow from this simple fact. It means that we do not measure the reflected energy density directly (as one might visuallze, for example, using the polychromatic and non-coherent sun as an fllumination source, derfving thus an analog of "reflectivity"). The monochromatic radar 1llumination makes the radar behave as an interferometer, whose input is a linear sum of complex amplitude signals, each of the form

$$
\left|\sigma_{0}(x, y)\right|^{1 / 2} e^{j \phi(x, y)} e^{j(z \pi f t+\theta)}
$$


Thus the iqput includes the (square root of) reflectivity, but also the phase $\phi(x, y)$ of each reflecting elemeni, which of course is directly affected by the (accidenial) distance and aspect angle between that reflectnr and the radar. The phase is a mixed blessing, for it allows "synthetic aperture" resolution (Brown and Porcello, 1969) (beam sharpentng) to occur; it also gives rise to "speckle" (described below).

iv) Whereas the probe of the scene is at complex (microwave) amplitude, the image is in terms of amplitude magnitude squared. Thus, in numerical terms, the image $\hat{\sigma}_{o}(x, y)$ is a real non-negative variable in contrast to the radar's observation in the scene, which is in terms of complex amplitude. (This seeming non-linear tranzformation is the key to the radar princlples of "conservation of energy".)

v) A SAR works because the zadar's motion imposes a structure on the phases of the received signa!s that can be used to "focus" the resulting lmagery to a specific resolution (Brown and Porcello, 1969; Harger, 1970; Raney, 1983). Once this is accomplished, then the phase information becomes irrelevant, and now the disadvantages of the remaining phase structure (speckle) become lmportant. Speckle can be reduced (at the expense of resolution - see below) in elther of the two dimensions, range or azimuth, by one of two linear techniques, frequency domain filtering (subapertures) (Bennett and McConnel1, 1980; Porcello, 1976) or by adjacent cell averaging (Zelenka, 1976). These two techniques, frequency douain and image domaln, are mathematically equivalent for stationary inputs and SAR type systems (Raney, 1983), an important consideration for users who may have to deal with pre-formed imagery. (Non-1inear speckle reduction teciniques may also be employed, but are not of interest in this paper.)

v1) Final1y, ail of the above deals with systems that present 1magery in $\hat{\sigma}_{0}(x, y)$ form, $1 . e$. , amplitude squared. There are systems, such as the MDA G-SAR processor, that (a) do a square-root or other amplitude mapping, and (b) perform a "most significant bit" or other automatic gain control function, both with the intent to improve Image cosmetics or data volume compression. It is important to note that the considerations of this paper apply to the "unscaled amp11tude square" image data format. For those wishing to persue experiments in this area, elther access to such data is essential, or suitable transformations are required.

Given all of the above, there exists a "model" of a SAR system that Inccrporares these character $18 \mathrm{ctcs}$, In terms of a generalized quadratic filter theory (Raney, 1983). In this language, a SAR is described (for either the range or the azimuth channel) as a simple sequence of operations

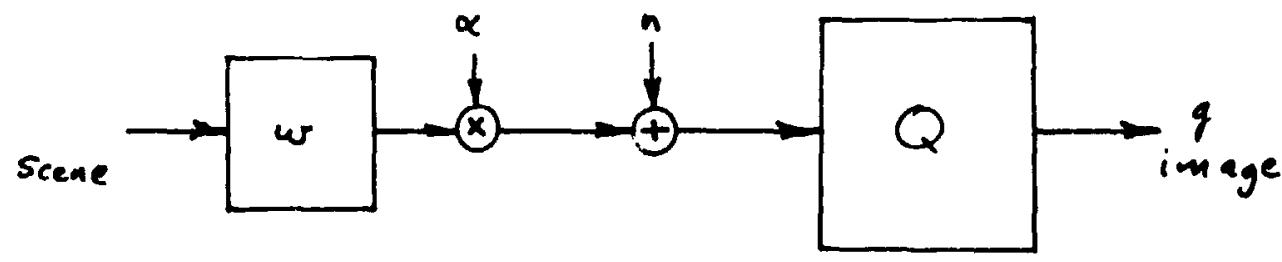


... In which the input is in complex amplitude, the range coding or azimuth Doppler modulation is represented by the pre-filter $w$, multiplicative random phase perturbation, additive (complex receiver) noise $n$ enters, the data 1 s focussed, amplitude squared, and speckle smoothed $(Q)$ to arrive at the image $g$.

For the following, we assume that the systom 1s "1near", but not "perfect". There may occur focus errors, or variations in the coherence of the processor (1.e. Intentional speckle smoothing) or in the scerie (unfintentional, resulting from sea surfare motion). We will be 1 in:erested in measures of $g$ (the 1mage) as they relate to propertles of the scene and the processor.

The system 1s linear in a speclal sense. A SAR, 11ke other types of radars, includes filters linear in complex amplitude (pre-detection or coherent 1ntegration), square law detection, and 1mage smoothing (post-detection oz nor-coherent integration, 1.e., "multi-look1ng" ir popular SAR term(nology). For such partlally coherent systems, the input/output relationshif may be expressed in terms of a modulation transfer function ( $0^{\prime} N e 111,1963$ ) which is linear in intensity (spatial reflestivity density). This is valid, no matter the degree of partial coherence of the (radar) system or the scene (Raney, 1983).

\section{ITPULSE RESPONSE}

The response of a SAR to a small specular polnt scatterer such as a corner reflector glves rise to an image pattern that is of fundamental importance in system characterization, analogous to the "point-spread function" of non-coherent optics (0'Ne111, 1963). The impulse response is the classic test signal for radars.

Let the Impulse response be represented by $g_{f}(u)$, a non-negat Ive function with units of voltage squared. is well behaved impulse response will be sharply peaked

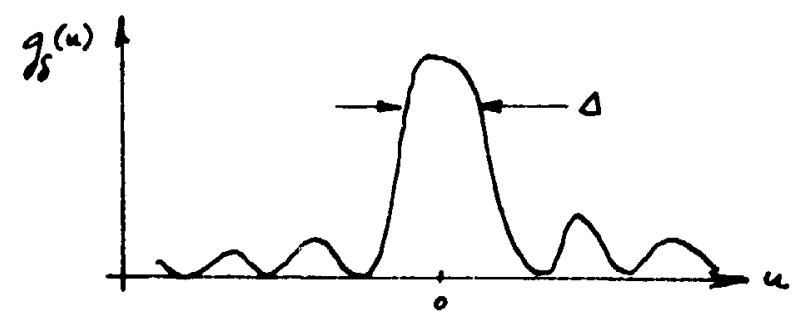

.... has a width $\Delta$ at the half-power level, and has "reasonable" side lobes. The width $\Delta$ is (loosely) referred to as the resolution of the radar, in either the range or azimuth dimension.

The following properties may be proven for the impulse response: 
1) The energy $\int g_{\delta}(u) d u$ in the response 1 s constant in the face of focus variations in the SAR (processor), random phase perturbations In the reflector, and for djffering degrees of nor. cunerent integration (mult1-1ooking). There is no coherent gain on the mean reflectivity of a point (coherent) target. This is the conservation of energy principle for polnt targets.

11) The fully coherent correctly focussed impulse response has width $\Delta$, which is the minimum width obtainable from the system. The inverse width $\left(\Delta_{1}\right)^{-1}$ is a measure of the effectfve system bandwidth In the pertinent channel, sultably scaled from spatial coordinates to Hertz. Focus errors, or partial coherence in the point target, result in broadening of the impulse responses and reduction of its peak.

111) For a given system differing amounts of non-coherent integration may be employed in the processor. Increased non-coherent integration degrades resolution. For $N$ statistically independent looks, the corresponding impulse response width $\Delta_{\mathrm{N}}=\mathrm{N} \Delta_{1}$.

iv) It follows that for an N-look response, the peaik is reduced $\mathrm{N}$-fold. This occurs because specular scatterers maintain their coherence as the radar observes them, so that the coherent gain due to processor focus is reduced as less of the signal is used coherently. Note that the famous coherent gain of a SAR is applicable to the peak value of reflectivity of a single coherent scattering centre (In one resolution cell), and is dependent on scene and system coherence.

v) As an obvious but important generalization, it follows that for a specular scatterer, there is very high correlation of the response between any two looks of a mult1-look set.

v1) In the event that there are deterministic phase perturbations on a point scatterer, azimuth shift (proportional to the linear phase term) and azimuth defocus (proportional to the quadratic phase error) plague the affected impulse respc nse (Raney, 1971). The radar processor may be retuned to match these perturbations, but at the expense of becoming mis-matched to all other signals in the processed fleld. (It follows that if there are a variety of different shift and focus perturbations affecting various scatterers in the scene, they cannot all be optimally processed simultaneously.)

\section{RESPONSF. IO DISTRIBUTED SCELES}

For many remote sensing applications, the response of a SAR to distributed scenes $1 \mathrm{~s}$ of more 1nterest than the polnt target response. One can show (Raney, 1983) that the input/output relationship is $\sigma_{0}(x, y)=g_{g}(x, y) \star \star \sigma_{0}(x, y)$ where $* \star$ denotes convolution on the $x$ and $y$ coordinates respectively and $g,(x, y)$ is the approprlate 1mpulse response of the SAR. The following properties are satisfled: 
1) The average value of the output, for noninally constant input, is a constant, Indenendent of system focus, system coherence, or scene coherence, assuming that all avallable data is used in each case. This is the most fundamental feature of the system, the principle of conservation of energy. It means that there $1 \mathrm{~s}$ no coherent gain by the radar or processor for the mean reflectivity of diffuse distributed scenes. Furthermore, between multi-look sets, relative gain can be normalized since tctal average response is not a function of the number of looks.

11) From 1) 1t follows that a SAR's response to a (Gaussian) distributed scene relative to recelver nolse is not a function of processing. Thus, for a given radar and scene, the SNR is constant as processor focus and coherence are varied, assuming that all the avallable data is used.

111) The two-dimensional Fourler transform of $g_{f}(x, y)$, written as $\tilde{g}_{g}(\omega, \lambda)$, is the modulation transfer function (MTY) of the system. If the system focus incorrect, partial coherence is used in the processor, or if there is loss of temporal coherence in the scene, then the width of the MTF is reduced, thereby limitirg the ability of the system to 1mage scene deta1l (Raney, 1983, 1980). This is of central importance in the response of a SAR to distributed dynamic phenomena, such as ocean reflectivity. It has the heavy consequence that the appropriate impulse response for the SAR may not be the same for all parts of the image simultaneously.

\section{SPECKAE COISIDBRATIONS}

The output of the system is deeply modulated even for nominally constant input $\sigma_{o}(x, y)$. Th1s phenomenon is known as speckle (Zelenka, 1976; Porcel1o, 1976; Bennett and McConne11, 1980), and 18 a natural consequence of coherent lllumination by the radar of a Gauzsian scene. (By definttion, a Gaussian scene is one in which, for each (nominal) resolution cell, there are many effective scatterers of statisticly Independent arplitude and phase.)

1) To first and second order, speckle statistics (for a uniform Gaussian distributed scene) are not a function of system focus.

11) Speckle statistics are not a function of scene temporal coherence. Thus, for Gaussian scattering, one cannot use speckle measures to estimate scene coherence.

111) For a Gaussian random Input, the saveral "looks" separated by a mult1-10ok processor are largely uncorrelated. The correlation properties observed are a measure of the bandwidth and frequency welghting of the SAR/processor combination.

1v) One effective measure of speckle 18 its variance. The amount of non-coherent integration in the processor, that $1 \mathrm{~s}$, the effective number of statisticaliy independent looks $N$, may be estimated by the rat 10

$N=\mid$ mean value $\hat{\sigma}_{0} \mid 2 /$ variance $\hat{\sigma}_{0}=(\mathrm{VMS})^{-1}$

for a nominally uniform region of a given acene. 
v) As a consequence of the preceeding four properties, speckle can be used to estimate the potential resolution of a SAR/processor. The nominal 1mpulse response of the radar is closely approximated by the square root of the speckle covarlance function for correct focus and scene coherence. However, speckle cannot be used to estimate actual SAR performance agalnst part1cular (possibly dynamic) objects unless focus and scene coherence can be (independently) ascertained as correct for the dynamics of those objects.

v1) There is evidently a direct trade-of: between resolution $\left(A_{N}\right.$ proportional to $N$ ) and speckle reduction (variance inversely proportional to $\mathrm{N}$. This can he stated as the principle of conservation of confustun

$$
\int\left[R_{g}(x)-\xi^{2}\right] d x=\text { constant }
$$

tndependent of the degree of partial coherence, where $R_{g}(x)$ is the spatial correlation function, under the assumption of uniform Gaussian input.

vi1) In the event that the scene is not Gaussian at the nominal resolution cell level, then these propertles do not necessarlly hold. In part:1cular, if there are dominant scattering centres, then 1mage behavior w11l be described more appropriately by articles in Section 4 above, even if ti. si-called image resembles speckle in appearance.

\section{OBSERVATIONS ANID DMPLICATIONS}

The properties of SAR lmaging behavior introdur d above should be incontrovertible. Any observation, theoretical or experimental, that purports to "explain" the content ot SAR ocean liugery, or to go even further and to "explain" the scattering mechanism, must be consistent: with these principles.

There seem to be two general issues in active discussion in the theory of SAR ocean wave 1maging: Gaussian versus non-Gaussian scattering; and the causes (and possible remedy) of azimuth directional spectral narrowlng (Hasselmann et al., 1984). Therefore, it would be helpful to organize the foregolng SAR facts of 11 fe accordingly.

Table 1 compares the response of Gaussian scatterers and a specuiar scatterer for eleven measurable SAR image properties. These propertles in turn are organlzed into two groups, Generai Considerations, having to do with a nominally uniform average reflectivity (of which a wheat fleld is the classic example), and Two-scale Considerations, for which there 18 assumed a low (spatial) frequency modulation of the reflectivity, as by a swell or more fully developed sea. The Table is filled in under the assumption that the SAR azimuth response 18 of interest. 
TABLE 1

COAPARISON OF IMAGE PROPERTIES

\section{GADSSIAN SCATTERIMG SPBCULAR SCATTERER}

\section{General Cons1derations}

1. High correlation between looks (azlmuth sub-aperture filtering)

No

Yes

2. Average 1mage (Intensity)

dependent on

$\mathrm{N}$-look processing

No

No

3. Peak values of Image

(Intensity) dependence on $\mathrm{N}$

$\mathrm{N}^{-\frac{1}{2}}$

$N^{-1}$

4. Focus sensitivity

(uniform reflector)

No

Yes

5. Image senslt1vity to

scene coherence time

No

Yes

6. Fourier trans form of

"1mage" a measure of

SAR/processor (resolution) ${ }^{-1}$

No

Yes

7. Fourier transform of

"1mage" a measure of

SAR/processor bandw1dth

Yes

No

Two-acale Considerations

B. Velocity bunching

Yes

Yes

9. Velocity spreading

Yes

No

10. Focus sensitivity

Weak

Strong

11. Coherence time IImftation

Yes

Yes 
The 1ssue of Gaussian versus non-Gaussian scattering is an Important one, both technically and philosophically. From a technical polnt of view, there is ample evidence that non-Gaussian scattering 18 characterlstic of sea radar return (Trunk and George, 1970; Barkeshl1 and Moore, 1983), and Indeed for many applications it is the reflectivity characteristic of central concern, as in target deiection (Trunk and George, 1970), or in explaining the differ ince between alrborne and tower based scatterometer results (Barkesh11 and Moore, 1983). It seems well established that as the nominal resolution cell size decreases, the importance of "spikey" non-Gaussian reflectivity elements increases (Trunk, 19,2, and especially Jakeman and Pusey, 1976).

There are various "explanations" for non-Gaussian scattering. Most of these explanations are based on statistics that are closely related to the expected result of a very small average number of effectlve scatterers per resolution cell, where this value ranges from 0.1 r less (Jakeman and Pusey, 1976) to (1esg than) 5, the accepted inreshold for Rayleigh - hence Gausslan - scattering. For this reason, in Table 1 the non-Gaussian case is represented by a single (dominant) specular scattering centre in a resclution cell. There may or may not be such a scatterer in an adfacent cell in a typical sltuation, indeed usually not. Hence the point target propertles of SAR response apply to such a case.

For Gaussian scattering, it is assumed that there are "many" effective scattering centres per resolutiun cell.

As an aside, it is important to note that for a SAR, the number of effective scatterers per resolution cell is the spatial scale of interest, not the Instantaneous field of view (antenna widtn by pulse length) of the radar.

From a philosophical point of $v 1$ tw, the existence of and differences between Gaussian and non-Gaussian scatterers lis important in that agreement on the veracity or significance of theoretical or experimental results is impossthle unless assumptions about the underlying scattering, elther explicitly presented or implicitly employed, are clearly explored and consistently followed. Fron the point of view of this wrtter, most of the controversy in the "focus" area 18 potentially resolvable if $f$ irst there would be agreement on the type of scattering being considered.

Finally, some remarks on the nature of "a specular scaterer". It 10 obvious that a solld corner reflector 18 an example of a opecular scatterer, as 18 a facet whose plane 18 orthogonal to the radar range 11ne. It is likely that instances of coherent specular reflertion arlse from such geometrics accidentally simulated by the sea surface. Likewlse, point scattering by cusp or other surface discontinulty could provide a source of specular reflection. 
There is another source of "specillar" reflection that $s$ " $m$ s to have been overlooked to date; Bragg scatter1ng. Whereas Bragg scatte:1ng, the geometry in which the radar wavelength profected onto a wavy surface picks out preferentially the matching surface spatial frequency, is usually taken to be the epitomy of raussian scattering. (Hasselmann et al., 1984), it is by definition just the opposite! Bragg scaitering, for one set of resonant scatterers in a local region, results in a coherent specular signal. It is only if there is an ensemble of many such Bragg scattering cells in nne resolution cell that Gaussian statiatics again apply. The modelling issue then reduces to representation of the expected size of a Bragg region as compared to a radar resolution cell.

Differentiation between these two specular r flection concepts should be possible experimentally as they are modulated by quite different portlons of Lie crean Doppler spectrum. The first typlcally move at nominal phase speed of the longer waves, whereas the second are dominated by orbltal advection, hence much more slowly.

Irrczardless of "the cause" of specular events in BAR ocean Imagery, they do extst. Their onservable properties are in many cases different from proper Gaussian scattering. Search for and observation of these features is worthwhlle.

Turn now to the considerations of the Table. The first group (:tems 1-7) apply directly to a nominally ldealized scene, and may $b$.

visualized as beling analogous in the unlform Gaussian scattering case to determination of system response by white random nolse or in the specular scatterer case to the optical "point spread function" (O'Ne111, 1963).

It $1 \mathrm{~s}$ of central importance to this discussion that unlike convent lorial imaging $c$ : s"Btems analysis using purely non-coherent ll lumination, for partially coherent systems there is not a one-to-one equivalence between frequency domain, and $t$ ime donaln norms. Independent measures of frequency struccure (e.g., bandwluth) and temporal structure (e.g., coherence) are requlred. One purpose of the Table is to sugges: approaches to this question. In this sense, the first seven items carry over directly and Impact the final four items.

The Two-scale Considerations are meant to be those of $f$ irst order relevance under the assumption that one 18 attempting to "1mage" azimuth waves, and thus to understand the azimuth wave spectral response of a SAR.

In order to get a bit more depth into tise implications of the Table, consider an experlmeni using existing data. We need to have examplea of SAR ocean imagery with different qualities of azimuth waves visible. There shuuld also be avallable a control, an example of Imagery (frow the same radar and processor) thac Includes a large random fleld, and if possible at least one point reflector. The experimental procedure 1 s to perform on both the control and the sea Images the serles of tests sugested in the Table. From the Control, general considerations $1,2,3,4,6$ and 7 may be verified. The sane measuret: hould then be perforned on the ses images, wth the results used to clasify regione of ses seatterlis as doninantly Gausian or 
specular.

For example, it has been observed that the apparent speckle in certain SEASAT scenes is elongated in azimuth by several resolution cells. This feature has been called "streakle" $\star$, a most descriptive terminology. The occurrence of streakle seems to be correlated with reduction of the azlmuth bandwith of asooclate directional spectra. The streakle events should be processed according to the tests of Table 1. From this one may conclude whether or not they correspond to specular or Gaussian scattering.

Again, one may search for focus sensitivities. Certain investlgators have reported rinding focus dependence in SAR data. In the context of the Table, this corresponds to 1 tem 10 , in which there 18 a value fudgement requlred. The case may be strengthened, by subjecting the area in question to the test of 1 tem 1 . for those areas in which both strong focus dependence $1 \mathrm{~s}$ found and specular correlation between looks is satisfled, then more presumptive steps (such as wave helght estimat!on) can be hazarded.

It is true that an ensemble of scatterers, or a polnt scatter, should they have a Doppler (linearly changlng phase) component will suffer an azimuth position shift (item 8 in the Table). This is difficult to observe confidently on the ocean, however, due to the complexity of the full spectrum of motions present.

In the event that the sea spectrum 1s nearly pure swell, as is the case for ocean waves in a fleld of floating 1ce, and the problem of scattering coharence time 18 avolded, then the velocity bunching mechanlsm may be directly obseived (Raney, 1981). There are interesting questions that have to do wth identifyling the cause of loss of azimuth wave sensitivity in is SAR. Perhaps the lssue could be addressed by observation of a wivi field as it propagates from open water Into an ice covered reglon, progressively attenuating the higher frequency portion of the wave epectrum. Again, any experimental cbservations should be verified by a control frame of SAR data, and fudiclous use of the measureable norms of Table 1.

\section{7. conclosions}

This paper has attempted to present and tabulate lmaging properties of a SAR that are facts of life. These have been selectively explored as they apply to the ocean imaging problem.

It 1s suggested that wost SAR ocean laggery has gropert!es $a^{-}$both Gaussian and specular ocattering. Exieting theories and experimental obervatione are controversial largely due to inconsistencles in the assumed scatterin mechanisa. Logical approach to the problem requires that the rules of SAR lange formation be utilized as guldance to localize and interpret ocean laagery phenomena. An experimental p.ocedure 1s suggested to reallze this goal. 
Barkeshl1, S. and R.K. Hoore, 1983; "Interactiun of the Radar Waves with the Caplllary Wave on the Ocean", Untversity of Kansas. Remote iensing Laboratory Report RSL TR 419-1.

Bennett, J.R. ars P.R. McConre11, 1980; "Considerations in the Desigr of Optimal Multilook Processors tor lmage Quality", Proceedings of the 3rd SEASAT-SAR Forkshop on SAR Image Juality, Frascati, Italy, (ESA SP-172) PP. 5-12.

Brown, W.M. and L.J. Porcel10, 1969; "An Introduction to Synthetic Aperture Radar", IEEE Spectrum 6, pp. 52-62.

Harge, R.0., 1970; Synthet1c Ayerture Radar systems: Theory and Design. Academic Press, New York,

Hasselman, K., et al., 1984; "Theory of SAR Ccean Wave Imaging, A Marsen View", Journal of Geophysical Resea:ch (in publication).

Jakeman, E. and P.N. Puscy, 1976; "A Model for Non-Rayle1gh Sea Echo", IEEE Trans. Antennas and Propagation, Vo1. AP-24, No. 6.

O'Ne111, E.L., 1963; Introduction to Stat'st1cal Optics, Addisou-Wesley, Reading, Mass.

Porce11o, L.J. et al., 1976; "Speckle Reduction in Synthetic Apertire Radars", Journal of Optical Soclety of America, Vol. 66, No. 11, pp. 1305-1311.

Rancy, R.K., 1971; "Synthet1c Aperture Radar and Moving Targets", IEEE Trans. on Aerospace and Electronic Systems, AFS-7, Pp. 499-505.

Raney, R.K., 1980; "SAR Response to Partially Coherent Phenomena", IEEE Transactions on Antennas and propagation, Vol. AP-28, No. 6, pp. 777-787.

Taney, R.K., 1981; "Wave Orb1tal Veloc1ty, Fade, and SAR Response to Azlmuth Waves", IEEE Journal of Oceanic Engineering, Vol. OE-6, No. 4.

Raney, R.K., 1983; "Transfer Functions for Partially Coherent and SAR Systems", IEEE Transactions of Aerospace and Electronlc Systems, 19, No. 5 .

Trunk, G.V. and S.F. Gecrge, 1970; "Detection of Targets in Non-Gaussian Sea Clutter", IEEE Trans. Aerospace and Electronic Systems, Vol. AES-6, No. 5, pp. 620-628.

Trunk, G.V., 197?; "Radar Properties of Non-Rayle1gh Sea Clutter", IEEE Trans. Aerospace and Electronic Systems, Vo1. AES-8, No. 2, pp. 196-204.

Zelenka, J.S., 1976; "A Comparıson of Continuous and Discrete Mixed Integrator Processors", Journal of Optical Soclety of America, Vol. 66, No. 11, pp. 1.295-1304. 